\title{
SiRNA of Frizzled-9 suppresses proliferation and motility of hepatoma cells
}

\author{
TATSUYA FUJIMOTO, MINORU TOMIZAWA and OSAMU YOKOSUKA \\ Department of Medicine and Clinical Oncology, Graduate School of Medicine, \\ Chiba University, 1-8-1 Inohana, Chuo-ku, Chiba City, Chiba 260-8670, Japan
}

Received May 12, 2009; Accepted July 9, 2009

DOI: 10.3892/ijo_00000400

\begin{abstract}
Frizzled (Fz), a receptor of Wnt ligands, plays key roles in liver carcinogenesis. Its expression was analyzed as part of a search for a target of molecular therapy for hepatocellular carcinoma (HCC) and hepatoblastoma (HB). Fz genes were analyzed by RT-PCR in HCC cell lines HLE, HLF, PLC/PRF/5, Huh-7 and Hep3B, HB cell lines Huh-6 and HepG2, HeLa cells, human normal fetal and adult liver. We transfected PLC/PRF/5, HLE, Huh-6, and HeLa cells with Fz9-small interfering RNA (Fz9-siRNA). Five days after transfection, cell proliferation was analyzed by MTS assay and cell motility by wound assay with H\&E staining. Subsequently, the expressions of cyclin D1 and caspase-3 were analyzed by Western blot analysis. Fz9-siRNA decreased the expression of Fz9 gene in all cell lines. MTS assay showed that Fz9-siRNA significantly suppressed cell proliferation and cell motility in all cell lines. The expression of cyclin D1 was also suppressed by Western blotting. Cleaved caspase-3 did not appear and apoptosis was not observed in any of the cell lines tested. We demonstrated that Fz9 plays an essential role in carcinogenesis of $\mathrm{HB}$ and $\mathrm{HCC}$, concluding that Fz9siRNA could represent a useful therapeutic target for $\mathrm{HB}$ and HCC.
\end{abstract}

\section{Introduction}

The Wnt signalling pathway plays a crucial role in regulating cell growth and differentiation. Constitutive activation of the Wnt pathway causes abnormal cell growth and cancer (1-3). Frizzled (Fz) is a cell surface receptor that mediates the actions of Wnt ligands, and 10 members of the Fz family genes have been identified based on structural and functional homologies

Correspondence to: Dr Minoru Tomizawa, Department of Medicine and Clinical Oncology, Graduate School of Medicine, Chiba University, 1-8-1 Inohana, Chuo-ku, Chiba City, Chiba 260-8670, Japan

E-mail: nihminor-cib@umin.ac.jp

Key words: frizzled, siRNA, hepatocellular carcinoma, hepatoblastoma
$(4,5)$. Fz genes have been implicated in carcinogenesis and embryogenesis in previous studies. Their expressions are upregulated in gastric, colon, breast, and renal cell carcinoma, suggesting that Fz genes are involved in carcinogenesis (6-12).

Regarding liver carcinogenesis, $\mathrm{Fz}$ genes play a crucial role. Hepatocellular carcinoma (HCC) arises in liver of Fz7 transgenic mice (13). Fz3, Fz6 and Fz7 genes are upregulated in HCC tissues, compared with surrounding nontumor tissues $(14,15)$. However, because Fz3, Fz6 and Fz7 are expressed in surrounding non-tumor tissues, their clinically mediated inhibition may give rise to adverse effects. If a Fz gene would not be expressed in surrounding non-tumor tissues, it would be a good candidate for molecular therapy for HCC by inhibition of its activity. However, there is no literature on any $\mathrm{Fz}$ gene being expressed in tumor tissues while not expressed in surrounding non-tumor tissues. We thus analyzed the expressions of Fz genes of HCC cell lines, hepatoblastoma (HB) cell lines as well as HeLa cells, in an attempt to discover a novel therapeutic target.

\section{Materials and methods}

Cell lines and culture conditions. Human HB cell lines (Huh-6 and HepG2) and HCC cell lines (PLC/PRF/5, HLE, HLF, Huh-7 and Hep3B) were purchased from RIKEN Cell Bank (Tsukuba, Japan) and cultured in Dulbecco's minimum essential medium (DMEM; Sigma, St. Louis, MO) supplemented with $10 \%$ fetal bovine serum (FBS) (Trace Scientific, Melbourne, Australia) in $5 \%$ carbon dioxide at $37^{\circ} \mathrm{C}$ in a humidified chamber. Cells were spread onto $10-\mathrm{cm}$ dishes (Asahi Techno Glass, Funabashi, Japan) for Western blot analysis, 4-well chamber slides for wound assay (Iwaki, Tokyo, Japan), or 96-well flat bottom wells for proliferation assay (Asahi Techno Glass).

Reverse transcriptase-PCR. Total RNA $(5 \mu \mathrm{g})$, isolated with Isogen (Nippon Gene, Tokyo, Japan), was used for first-strand cDNA synthesis with SuperScript III and oligo (dT) following the manufacturer's instructions (Invitrogen Japan K.K., Tokyo, Japan). RNAs of human fetal whole liver and human adult whole liver were purchased from Takara Bio (Kyoto, Japan). Polymerase chain reaction (PCR) was performed with Taq DNA polymerase (Applied Biosystems Japan, Tokyo, Japan). PCR primers, annealing temperature, number of cycles, and the length of the amplified products were as 
below: Fz1 (GenBank accession no. NM_003505, 5'-AATG ACAAGTTCGCCGAGGAC, 5'-GCCAGGTGAAAATACT GTGAGTTGG; 59 ${ }^{\circ} \mathrm{C}$; 30 cycles, 206 bp), Fz2 (GenBank accession no. NM_001466, 5'-CAAGGTGCCATCCTAT CTCAGC, 5'-GTAGCAGCCCGACAGAAAAATG; 59 C; 30 cycles, 247 bp), Fz3 (GenBank accession no. NM_017412, 5'-AGAGAAGAACTGTCATTTGCTCGC, 5'-TCCTTGTG TCACTGTGGAAGCC; $53^{\circ} \mathrm{C} ; 30$ cycles, 255 bp), Fz4 (GenBank accession no. NM_012193, 5'-CAAGTGATTCTC CTGCCACAGC, 5'-CAACTCTCTCCAGTGTCCTCCATC; $57^{\circ} \mathrm{C} ; 30$ cycles, $270 \mathrm{bp}$ ), Fz5 (GenBank accession no. NM_003468, 5'-CCCTCATCCCCTAAGAGAGACAAAG, 5'-GCTGGCTGTGAAGAAGTTGCTG; 55 ${ }^{\circ} \mathrm{C} ; 30$ cycles, 230 bp), Fz6 (GenBank accession no. NM_003506, 5'-AGC AGCATCCATCTCCAGACTCTC, 5'-CTGAATGACAACC ACCTCCCTG; $57^{\circ} \mathrm{C}$; 30 cycles, 251 bp), Fz7 (GenBank accession no. NM_003507, 5'-AGACTTAGCCACAGCA GCAAGG, 5'-CGCCGTTATCATCATCTTCCTG; 58 ${ }^{\circ}$; 30 cycles, 287 bp), Fz8 (GenBank accession no. NM_031866, 5'-ATCCAAAGCAGATGCCATTGTC, 5'-AACACTGTGA AGGGGTGGGAAC; $59^{\circ} \mathrm{C} ; 30$ cycles, 137 bp), Fz9 (GenBank accession no. BC_026333, 5'-TCTTTGGAGAACCCCA CACACC, 5'-TGCTCACTTGCCTGACCTTGAC; $60^{\circ} \mathrm{C}$; 30 cycles, 148 bp), Fz10 (GenBank accession no. NM_007197, 5'-AAACGCTGGACTGCCTGATG, 5'-GCTTTTTTGTAA ATCCCACCGC; $58^{\circ} \mathrm{C} ; 30$ cycles, 217 bp), GAPDH (GenBank accession no. NM_002046, 5'-ACCTGACCTGCCGTCTA GAA, 5'-TCCACCACCCTGTTGCTGTA; 63ํㅜ 30 cycles, $246 \mathrm{bp})$.

SiRNA transfection. Transfection of siRNA of Fz9 (Fz9siRNA) (Invitrogen Japan K.K.) was carried out using Lipofectamine 2000 (Invitrogen Japan K.K.) and Opti-MEN (Invitrogen Japan K.K.), according to the manufacturer's protocol. Briefly, siRNA and Lipofectamine 2000 were diluted in Opti-MEN at room temperature for $5 \mathrm{~min}$, separately. Then the diluted siRNA and Lipofectamine 2000 were incubated for a further $20 \mathrm{~min}$ at room temperature for complex formation. The medium was aspirated from each dish or well, and the complexes were added to cultured cells. Transfection efficiency was analyzed as $\sim 80 \%$ in PLC/PRF/5 and HeLa cell lines, and 90\% in HuH-6 and HLE cell lines, respectively, with a fluorescein isothiocyanate (FITC)-labelled oligodeoxynucleotide (Invitrogen Japan K.K.) under this transfection condition (data not shown).

Cell viability assay. Freshly thawed cells were seeded onto $10-\mathrm{cm}$ dishes. When they reached sub-confluence, they were trypsinized, harvested, and spread onto 96-well flat-bottom plates (Asahi Techno Glass) at a density of 1000 cells per well. Following $24 \mathrm{~h}$ of culture in DMEM with $10 \%$ fetal bovine serum, transfection was performed at a final concentration of $200 \mathrm{nM}$ of siRNA in each well. As negative control, mock, Lipofectamine 2000 alone was used. Five days after transfection, 3-(4,5-dimethylthiazol-2-yl)-5-(3-carboxymethoxyphenyl)-2-(4-sulfophenyl)-2H-tetrazolium inner salt (MTS) assay was performed according to the manufacturer's instructions (Promega Corp., Tokyo, Japan). MTS is bioreduced by cells into a coloured formazan product that reduces absorbance at $490 \mathrm{~nm}$. The absorbance was analyzed with

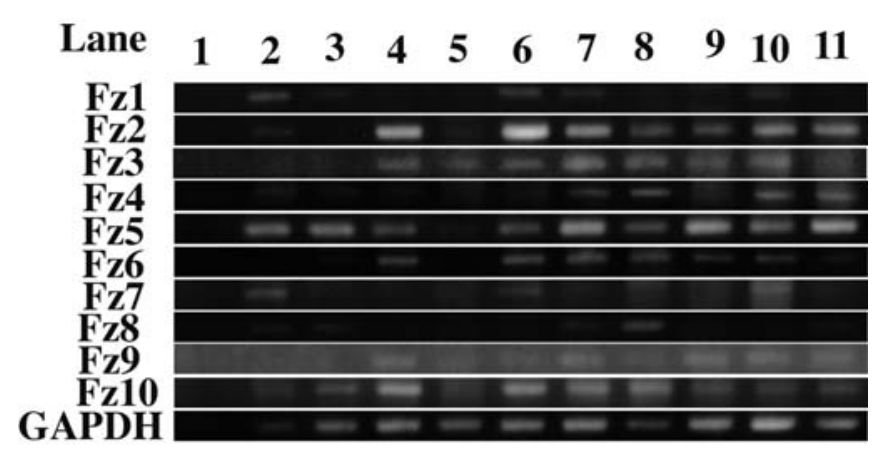

Figure 1. RT-PCR. Expression of human Fz genes was analyzed by RT-PCR with human fetal and adult liver, $\mathrm{HCC}$ and $\mathrm{HB}$ cell lines. A representative RT-PCR shows the expression of $10 \mathrm{Fz}$ genes in $\mathrm{H}_{2} \mathrm{O}$ (lane 1), human fetal whole liver (lane 2), human adult whole liver (lane 3), HeLa (lane 4), HLE (lane 5), HLF (lane 6), PLC/PRF/5 (lane 7), Huh-7 (lane 8), Hep3B (lane 9), Huh-6 (lane 10), HepG2 (lane 11). GAPDH was used as an endogenous control. Fz3 and Fz9 genes were expressed in $\mathrm{HCC}, \mathrm{HB}$, and HeLa (lanes 4-11), but not in normal fetal and adult liver (lanes 2 and 3).

a multiple plate reader at a wavelength of $490 \mathrm{~nm}$ with a Bio-Rad Model 550 microplate reader (Bio-Rad, Hercules, CA).

Western blot analysis. Cells grown in culture dishes were transfected at $\sim 70 \%$ confluence, at a final concentration of $200 \mathrm{nM}$ of siRNA. At 5 days after transfection, the cells were harvested for Western blot analysis. Protein $(20 \mu \mathrm{g})$ isolated from cultured cells was subjected to SDS-PAGE, and transferred to a nylon filter. The primary antibodies were polyclonal rabbit anti-human Fz9 (Lifespan Bioscience, Seattle, WA), polyclonal rabbit anti-human cyclin D1 (Cell Signalling Technology Japan K.K., Tokyo, Japan), monoclonal rabbit anti-human caspase-3 (Cell Signalling Technology Japan K.K.), and mouse monoclonal anti- $\alpha-$ tubulin antibody (Lab Vision, Fremont, CA). The second antibodies were HRP-linked anti-rabbit antibody (Amersham Bioscience, Tokyo, Japan) and HRP-linked anti-mouse antibody (Amersham Bioscience). Dilutions were 1:500 for the primary antibodies and 1:1000 for the second antibodies. The filters were reprobed with tubulin- $\alpha$. The specific antigenantibody complexes were visualized by enhanced chemiluminescence (Amersham Bioscience). The immunoreactive bands were normalized with tubulin- $\alpha$ and analyzed using Scion imaging software (Scion Imaging, Frederick, MD).

Wound assay. Cells were spread onto 4-well chambers (Iwaki). After $24 \mathrm{~h}$, transfection was performed at a final concentration of $200 \mathrm{nM}$ of siRNA in each well. Five days after transfection, sheets of cells were cut with a sterile razor. Two days later, cells were stained with hematoxylin-eosin and observed under a microscope. For each experiment, the number of cells migrating $>50 \mu \mathrm{m}$ of cut surface was counted in five different fields for each slide.

Statistical analysis. Cell proliferation was analyzed statistically with one-factor analysis of variance using Statview (version 5.0; SAS Institute Japan, Tokyo, Japan). A P<0.05 was accepted as statistically significant. 
(a)PLC/PRF/5 Fz9-

\begin{tabular}{|c|c|}
\hline Lane & Mock siRNA \\
\hline Fz9 & $24 \mathrm{k}=-24 \mathrm{kDa}$ \\
\hline $\begin{array}{l}\text { Fz9-siRNA } \\
\text { /mock }\end{array}$ & 0.18 \\
\hline
\end{tabular}

(c)Huh-6

Fz9-

Lane

Mock siRNA

Fz9

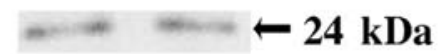

Fz9-siRNA

/mock

Tubulin $\alpha$

\subsection{3}

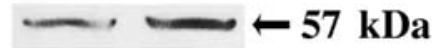

(b)HLE

Lane

Fz9

Fz9-siRNA

/mock

Tubulin $\alpha$
Fz9-

Mock siRNA

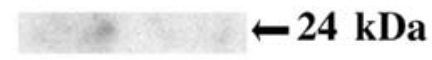

0.42
(d)HeLa

Lane

Fz9

Fz9-siRNA

/mock

Tubulin $\alpha$

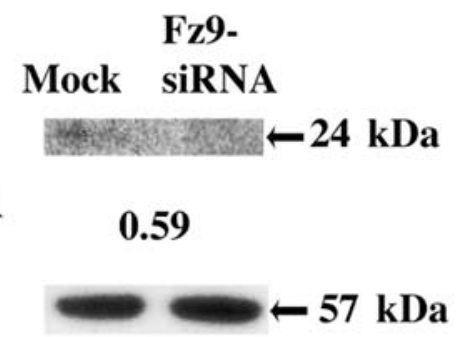

Figure 2. Western blot analysis of Fz9. The expression of Fz9 gene in PLC/PRF/5, HLE, Huh-6 and HeLa cells was examined by Western blotting with $20 \mu \mathrm{g}$ of isolated protein 5 days after transfection of Fz9-siRNA. The expression of Fz9 gene was normalized against tubulin- $\alpha$, a loading control, and the ratio of Fz9-siRNA to mock was calculated as Fz9-siRNA/Mock. Fz9 was down-regulated in cells transfected with Fz9-siRNA.

\section{Results}

Expression of $\mathrm{Fz}$ genes. The expressions of $10 \mathrm{Fz}$ genes were analyzed by RT-PCR in human fetal and adult liver, and in HCC and HB cell lines. Fz3 and Fz9 genes were expressed in all HCC cell lines, HB cell lines and HeLa, but they were not expressed in normal fetal and adult liver (Fig. 1). Other Fz genes were expressed in normal fetal and adult liver.

Suppression of cell proliferation. First, we focused on Fz3, as it was expressed in HCC cell lines consistently according to a previous report (15). To investigate the role of Fz3 in cell proliferation, we transfected PLC/PRF/5, HLE, Huh- 6 and HeLa cells with Fz3-siRNA. Five days after transfection, cell viability was analyzed by MTS assay. Fz3-siRNA did not suppress cell proliferation (data not shown). Fz9-siRNA was then transfected, and the expression of Fz9 was analyzed by Western blotting (Fig. 2). The expression level of Fz9 of cells transfected with Fz9-siRNA was observed to decrease compared to the corresponding mock in all cell lines (Fig. 2). MTS assay showed that Fz9-siRNA significantly suppressed the proliferation of all cell lines (Fig. 3).

Wound assay. Wound assay was performed to unveil the role of Fz9 in cell motility (Fig. 4). Fewer cells migrated $>50 \mu \mathrm{m}$ in PLC/PRF/5 cells than mock, while no cells migrated $>50 \mu \mathrm{m}$ in HLE, Huh-6 and HeLa cells, indicating that Fz9-siRNA significantly suppressed cell motility $(\mathrm{P}<0.05)$.

Western blot analysis. To determine the role of Fz9 in the cell cycle or apoptosis, the expressions of cyclin D1 and caspase- 3 were analyzed by Western blotting (Fig. 5). The levels of cyclin D1 after Fz9-siRNA transfection decreased in all cell lines. On the other hand, the expression levels of (a) PLC/PRF/5

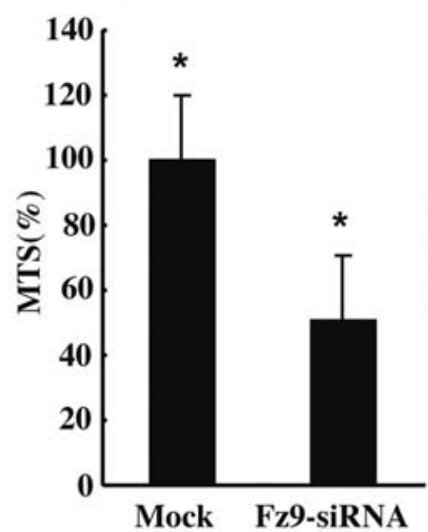

(c) Huh-6

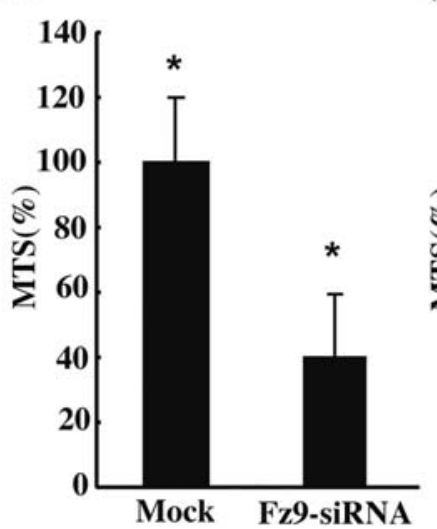

(b) HLE

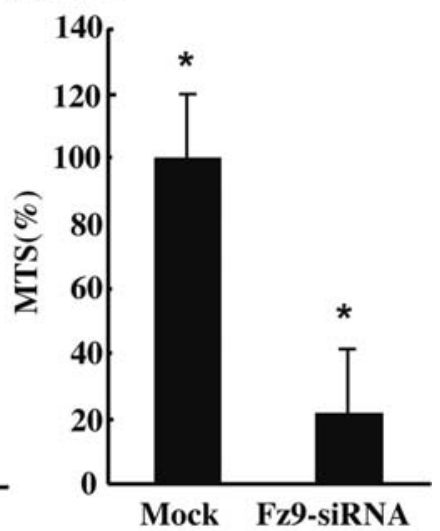

(d) HeLa

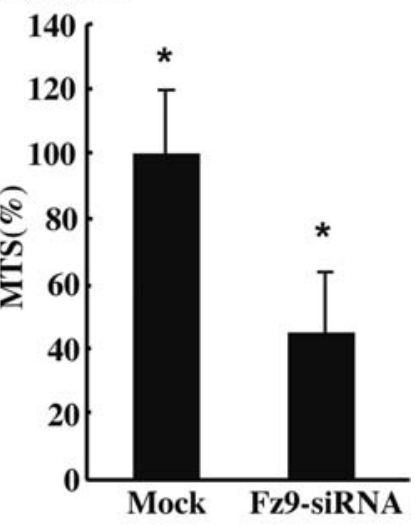

Figure 3. MTS assay. PLC/PRF/5, HLE, Huh-6, and HeLa, cells were transfected with Fz9-siRNA at a final concentration of $200 \mathrm{nM}$. Five days after transfection, cell viability was analyzed by MTS assay. Each value was normalized against the corresponding mock. Fz9-siRNA significantly suppressed the proliferation of all cell lines. ${ }^{*} \mathrm{P}<0.05$. 
A

(a) PLC/PRF/5

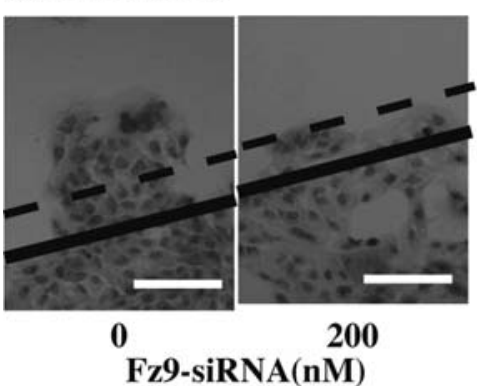

(c) Huh-6

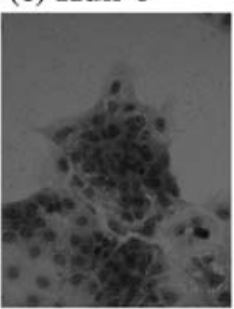

0

Fz9-siRNA(nM)

B

(a) PLC/PRF/5

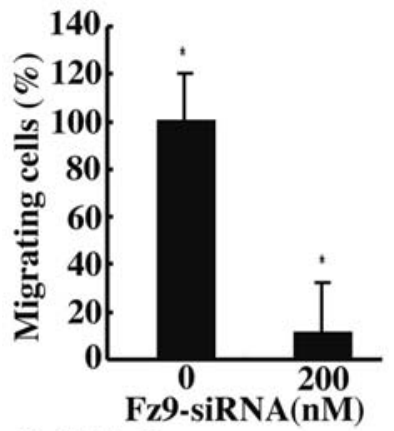

(c) Huh-6

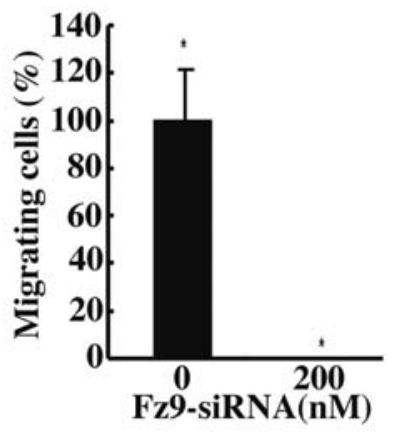

(b) HLE

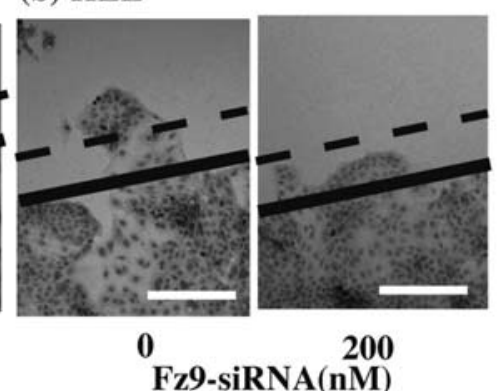

(d) HeLa

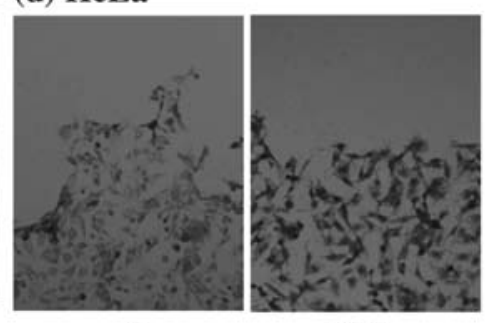

200

Fz9-siRNA(nM)

(b) HLE

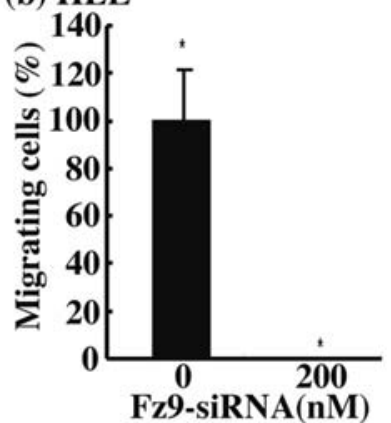

(d) HeLa

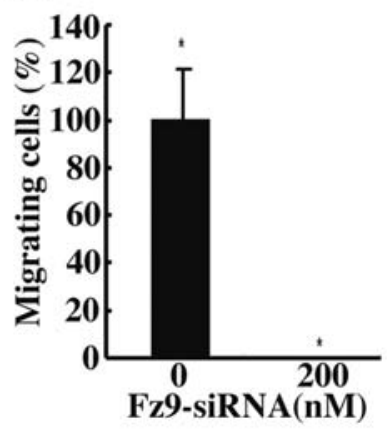

Figure 4. Wound assay. Wound assay was performed to analyze cell motility. Cells were transfected with Fz9-siRNA, stained with hematoxylin-eosin and observed under a microscope (A). The cells cultured with Fz9-siRNA and migrating $>50 \mu \mathrm{m}$ were counted, and normalized against the corresponding mock (migrating cell in B). Fz9-siRNA significantly suppressed cell motility in each cell line (B). (A) Original magnification x200; scale bar, $50 \mu$ m; (B) ${ }^{*} \mathrm{P}<0.05$.

17 and $19 \mathrm{kDa}$ of cleaved caspase-3, an essential element of apoptosis, did not appear. The expression levels of $35 \mathrm{kDa}$ of caspase-3, a non-cleaved form, did not change. No pyknotic cells were found in any of the cell lines transfected with Fz9-siRNA (Fig. 4A).

\section{Discussion}

Human Fz9 is expressed in the brain, testis, eye, skeletal muscle and kidney, but not in liver (16). Our RT-PCR also showed no expression of Fz9 gene in human adult liver, consistent with the previous report. This finding suggested that Fz9 played only a slight role in liver development. Since $\mathrm{Fz} 9$ is expressed in brain and skeletal muscle, its deletion causes musculoskeletal anomalies and neurological problems in Williams syndrome $(16,17)$. Fz9 expression in human astocytoma and glioblastoma was significantly higher than in normal brain (18). Interestingly, Fz9 is not originally expressed in astrocytes, and tumor cells acquired the expression of Fz9 (19). The expression level of Fz9 increases as tumor cells 
(a) PLC/PRF/5

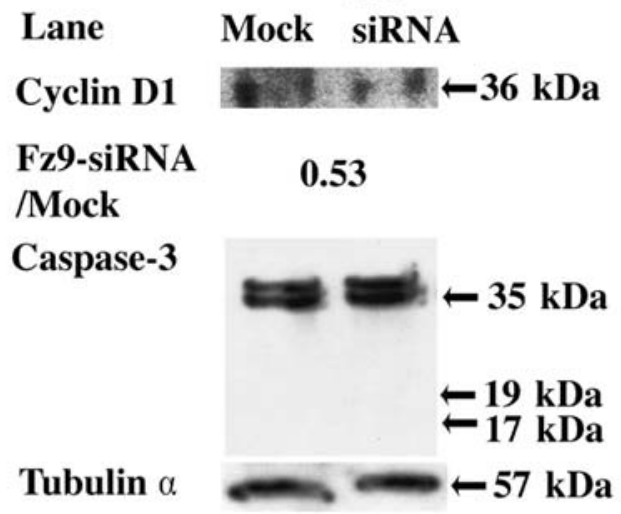

(c) Huh-6

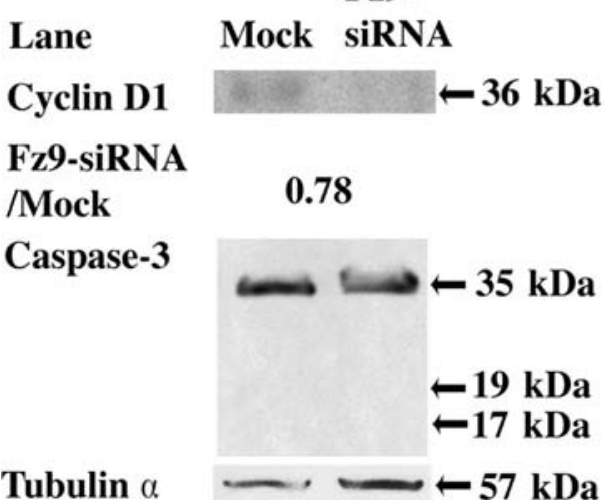

(b) HLE

Fz9-

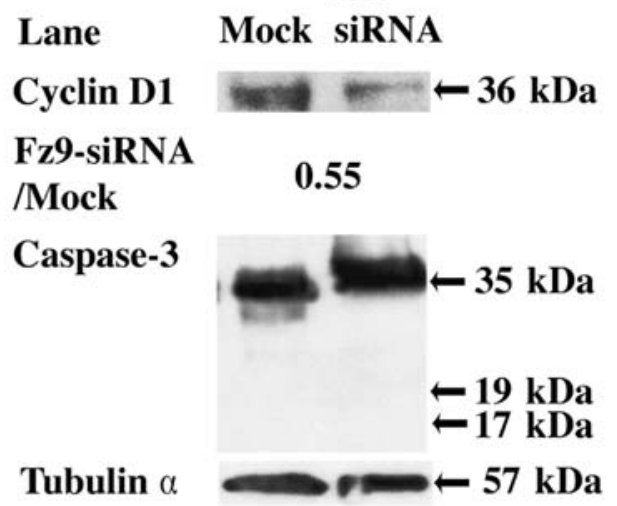

(d) HeLa

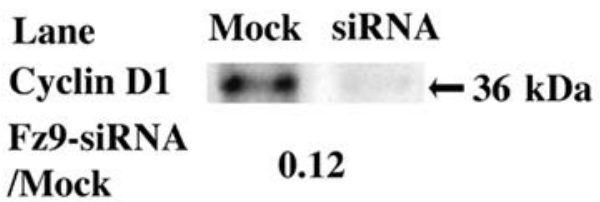

Caspase-3

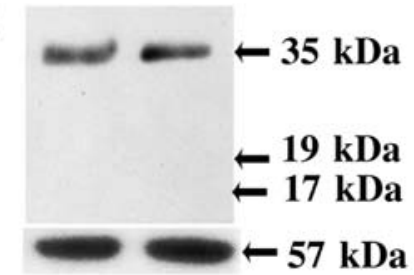

Figure 5. Western blot analysis of cyclin D1 and caspase-3. Effect of Fz9-siRNA on cell cycle and apoptosis. Western blot analysis was performed with cyclin D1 and caspase-3 in PLC/PRF/5, Huh-6, HeLa, and HLE cells transfected with Fz9-siRNA. Tubulin- $\alpha$ served as a loading control. Fz9-siRNA/mock, a ratio of the cyclin D1 expression level normalized against tubulin- $\alpha$ in cells transfected with Fz9-siRNA divided by that in mock. Fz9-siRNA suppressed the expression of cyclin D1. The expression levels of 17 and $19 \mathrm{kDa}$ of cleaved caspase-3, an essential element for apoptosis, were not detected in any of the cells.

become less differentiated. Increased expression of Fz9 is involved in the carcinogenesis of astrocytoma and glioblastoma, and the expression of Fz9 increased markedly in colorectal cancer compared to normal tissues (10). Our results showed that Fz9 was expressed in all cell lines, and indicated that Fz9 was involved in carcinogenesis, although the detailed mechanisms remained elusive. The biological significance of the result that Fz9 was expressed in HCC and HB cell lines still needs to be unravelled. MTS assay revealed that Fz9-siRNA significantly suppressed the proliferation of all cell lines. We then used Western blotting to examine the expression of cyclin D1, known to be deeply involved in the cell cycle (Fig. 5), and found it to be significantly down-regulated in all cell lines transfected with Fz9-siRNA. Subsequently, caspase-3, a target effecter of apoptosis, was analyzed. Its cleaved form was not detected at all, while the expression level of the $35 \mathrm{kDa}$ form was not changed. This indicates that Fz9 suppressed cell proliferation via the cell cycle, not through apoptosis. This hypothesis was supported by the result of $H \& E$ staining, that pyknotic cells were not observed (Fig. 4A).

A previous study reported that Fz3, Fz6 and Fz7 were upregulated in tumor tissues compared to non-tumor ones (15).
In our experiments, Fz6 and Fz7 were not expressed in any of the cell lines tested, while Fz3 and Fz9 were expressed in all cell lines. These discrepancies could be explained by the fact that our study did not use quantitative RT-PCR, possibly obscuring differences in expression levels. Fz3 was a good candidate for molecular therapy since it was expressed in all cell lines and not in fetal and adult liver in our study, and it was up-regulated in tumor tissues in the previous study. Fz3-siRNA failed to suppress cell proliferation. The reason is not known, but $\mathrm{Fz} 3$ might not be involved in cell proliferation in $\mathrm{HCC}$ and $\mathrm{HB}$.

Our result was the first concerning $\mathrm{Fz} 9$ being expressed in HCC and HB cell lines and not in fetal and adult liver. The previous report did not show its up-regulation. The reason for this discrepancy might be that tumor tissues contained noncancer cells, such as blood and endothelial cells, and the expression level of Fz9 might have been obscured. Our study was more accurate with pure cancer cell lines.

Fz7-siRNA suppressed cell proliferation (9). However, as it was expressed in fetal and adult liver in our results, it was not suitable for molecular therapy. On the other hand, because of its non-expression in fetal and adult liver, Fz9 was considered potentially useful. No adverse effects were 
anticipated if Fz9-siRNA were applied in a clinical situation, since Fz9-siRNA would not be expected to affect surrounding normal liver. Fz9-siRNA may thus prove to be useful in a clinical setting.

In conclusion, we demonstrated that $\mathrm{Fz} 9$ played an essential role in cell proliferation and motility of HB and HCC. It was considered possible that Fz9 gene could represent a promising target for novel molecular therapy in HCC as well as $\mathrm{HB}$.

\section{Acknowledgements}

This research was supported by a Research Grant-in-Aid for Scientific Research (C) from the Japan Society for the Promotion of Science (19590748).

\section{References}

1. Katoh M: WNT signaling pathway and stem cell signaling network. Clin Cancer Res 13: 4042-4045, 2007.

2. Katoh M: WNT signaling in stem cell biology and regenerative medicine. Curr Drug Targets 9: 565-570, 2008.

3. Nusse R: Wnt signaling and stem cell control. Cell Res 18: 523-527, 2008.

4. Bhanot P, Brink M, Samos CH, et al: A new member of the frizzled family from Drosophila functions as a Wingless receptor. Nature 382: 225-230, 1996.

5. Wang HY, Liu T and Malbon CC: Structure-function analysis of Frizzleds. Cell Signal 18: 934-941, 2006.

6. Kirikoshi H, Sekihara H and Katoh M: Expression profiles of 10 members of Frizzled gene family in human gastric cancer. Int J Oncol 19: 767-771, 2001.

7. Vincan E, Darcy PK, Smyth MJ, et al: Frizzled-7 receptor ectodomain expression in a colon cancer cell line induces morphological change and attenuates tumor growth. Differentiation 73: 142-153, 2005.

8. Vincan E, Darcy PK, Farrelly CA, Faux MC, Brabletz T and Ramsay RG: Frizzled-7 dictates three-dimensional organization of colorectal cancer cell carcinoids. Oncogene 26: 2340-2352, 2007.
9 Ueno K, Hiura M, Suehiro Y, et al: Frizzled-7 as a potential therapeutic target in colorectal cancer. Neoplasia 10: 697-705, 2008.

10. Nagayama S, Yamada E, Kohno Y, et al: Inverse correlation of the up-regulation of FZD10 expression and the activation of beta-catenin in synchronous colorectal tumors. Cancer Sci 100: 405-412, 2009.

11. Milovanovic T, Planutis K, Nguyen A, et al: Expression of Wnt genes and frizzled 1 and 2 receptors in normal breast epithelium and infiltrating breast carcinoma. Int J Oncol 25: 1337-1342, 2004.

12. Janssens N, Andries L, Janicot M, Perera T and Bakker A: Alteration of frizzled expression in renal cell carcinoma. Tumour Biol 25: 161-171, 2004.

13. Merle P, Monte S, Kim M, et al: Functional consequences of frizzled-7 receptor overexpression in human hepatocellular carcinoma, Gastroenterology 127: 1110-1122, 2004.

14. Merle P, Kim M, Herrmann M, et al: Oncogenic role of the frizzled-7/beta-catenin pathway in hepatocellular carcinoma. J Hepatol 43: 854-862, 2005.

15. Bengochea A, Souza MM, Lefrancois L, et al: Common dysregulation of Wnt/Frizzled receptor elements in human hepatocellular carcinoma. Br J Cancer 99: 143-150, 2008.

16. Wang YK, Samos CH, Peoples R, Perez-Jurado LA, Nusse R and Francke U: A novel human homologue of the Drosophila frizzled wnt receptor gene binds wingless protein and is in the Williams syndrome deletion at 7q11.23. Hum Mol Genet 6: 465-472, 1997.

17. Wang YK, Sporle R, Paperna T, Schughart K and Francke U: Characterization and expression pattern of the frizzled gene Fzd9, the mouse homolog of FZD9 which is deleted in WilliamsBeuren syndrome. Genomics 57: 235-248, 1999.

18. Zhang Z, Schittenhelm J, Guo K, et al: Upregulation of frizzled 9 in astrocytomas. Neuropathol Appl Neurobiol 32: 615-624, 2006.

19. Rawal N, Castelo-Branco G, Sousa KM, et al: Dynamic temporal and cell type-specific expression of Wnt signaling components in the developing midbrain. Exp Cell Res 312: 1626-1636, 2006. 